\title{
Some Traditional Mathematical Knowledge Management
}

\author{
Patrick D.F. Ion \\ Mathematical Reviews, AMS \\ ion@ams.org
}

What is mathematical knowledge and how can it be managed? There are not only differing views around on the management aspect but there is no real clarity or consensus on what mathematical knowledge is; indeed there are questions as to what knowledge is and what mathematics is. For the sake of definiteness I will adopt a particular stance from which to work, namely that aspect of organizing the knowledge of mathematics represented by Mathematical Reviews, for which I have worked since 1980. From that platform we can explore and speculate both historically and prospectively. Some new results of bibliometric and other machine-enabled examination of the mathematical literature will also be discussed. 